\title{
ALTERNATIVE PROOFS OF A FORMULA FOR BERNOULLI NUMBERS IN TERMS OF STIRLING NUMBERS
}

\author{
BAI-NI GUO AND FENG QI
}

\begin{abstract}
In the paper, the authors provide four alternative proofs of an explicit formula for computing Bernoulli numbers in terms of Stirling numbers of the second kind.
\end{abstract}

\section{INTRODUCTION}

It is well known that Bernoulli numbers $B_{k}$ for $k \geq 0$ may be generated by

$$
\frac{x}{e^{x}-1}=\sum_{k=0}^{\infty} B_{k} \frac{x^{k}}{k !}=1-\frac{x}{2}+\sum_{k=1}^{\infty} B_{2 k} \frac{x^{2 k}}{(2 k) !}, \quad|x|<2 \pi .
$$

In combinatorics, Stirling numbers of the second kind $S(n, k)$ for $n \geq k \geq 0$ may be computed by

$$
S(n, k)=\frac{1}{k !} \sum_{\ell=0}^{k}(-1)^{k-\ell}\left(\begin{array}{l}
k \\
\ell
\end{array}\right) \ell^{n}
$$

and may be generated by

$$
\frac{\left(e^{x}-1\right)^{k}}{k !}=\sum_{n=k}^{\infty} S(n, k) \frac{x^{n}}{n !}, \quad k \in\{0\} \cup \mathbb{N} .
$$

In $[5$, p. 536] and [6, p. 560], the following simple formula for computing Bernoulli numbers $B_{n}$ in terms of Stirling numbers of the second kind $S(n, k)$ was incidentally obtained.

Theorem 1.1. For $n \in\{0\} \cup \mathbb{N}$, we have

$$
B_{n}=\sum_{k=0}^{n}(-1)^{k} \frac{k !}{k+1} S(n, k) .
$$

The aim of this paper is to provide four alternative proofs for the explicit formula (1.4).

2010 Mathematics Subject Classification. Primary 11B68, Secondary $11 B 73$.

Key words and phrases. alternative proof; explicit formula; Bernoulli numbers; Stirling numbers of the second kind; Faà di Bruno formula; Bell polynomial.

Please cite this article as "Bai-Ni Guo and Feng Qi, Alternative proofs of a formula for Bernoulli numbers in terms of Stirling numbers, Analysis-International mathematical journal of analysis and its applications 34 (2014), no. 2, 187-193; Available online at http: //dx.doi.org/10.1515/anly-2012-1238." 


\section{Four alternative Proofs of the formula (1.4)}

Now we start out to provide four alternative proofs for the explicit formula (1.4).

Considering $S(0,0)=1$, it is clear that the formula (1.4) is valid for $n=0$. Further considering $S(n, 0)=0$ for $n \geq 1$, it is sufficient to show

$$
B_{n}=\sum_{k=1}^{n}(-1)^{k} \frac{k !}{k+1} S(n, k), \quad n \in \mathbb{N} .
$$

First proof. It is listed in [1, p. 230, 5.1.32] that

$$
\ln \frac{b}{a}=\int_{0}^{\infty} \frac{e^{-a u}-e^{-b u}}{u} \mathrm{~d} u .
$$

Taking $a=1$ and $b=1+x$ in (2.2) yields

$$
\frac{\ln (1+x)}{x}=\int_{0}^{\infty} \frac{1-e^{-x u}}{x u} e^{-u} \mathrm{~d} u=\int_{0}^{\infty}\left(\int_{1 / e}^{1} t^{x u-1} \mathrm{~d} t\right) e^{-u} \mathrm{~d} u .
$$

Replacing $x$ by $e^{x}-1$ in (2.3) results in

$$
\frac{x}{e^{x}-1}=\int_{0}^{\infty}\left(\int_{1 / e}^{1} t^{u e^{x}-u-1} \mathrm{~d} t\right) e^{-u} \mathrm{~d} u .
$$

In combinatorics, Bell polynomials of the second kind, or say, the partial Bell polynomials, $\mathrm{B}_{n, k}\left(x_{1}, x_{2}, \ldots, x_{n-k+1}\right)$ are defined by

$$
\mathrm{B}_{n, k}\left(x_{1}, x_{2}, \ldots, x_{n-k+1}\right)=\sum_{\substack{1 \leq i \leq n, \ell_{i} \in \mathbb{N} \\ \sum_{i=1}^{n} i \ell_{i}=n \\ \sum_{i=1}^{n} \ell_{i}=k}} \frac{n !}{\prod_{i=1}^{n-k+1} \ell_{i} !} \prod_{i=1}^{n-k+1}\left(\frac{x_{i}}{i !}\right)^{\ell_{i}}
$$

for $n \geq k \geq 1$, see [4, p. 134, Theorem A], and satisfy

$$
\mathrm{B}_{n, k}\left(a b x_{1}, a b^{2} x_{2}, \ldots, a b^{n-k+1} x_{n-k+1}\right)=a^{k} b^{n} \mathrm{~B}_{n, k}\left(x_{1}, x_{n}, \ldots, x_{n-k+1}\right)
$$

and

$$
\mathrm{B}_{n, k}(\overbrace{1,1, \ldots, 1}^{n-k+1})=S(n, k),
$$

see $[4$, p. 135], where $a$ and $b$ are any complex numbers. The well-known Faà di Bruno formula may be described in terms of Bell polynomials of the second kind $\mathrm{B}_{n, k}\left(x_{1}, x_{2}, \ldots, x_{n-k+1}\right)$ by

$$
\frac{\mathrm{d}^{n}}{\mathrm{~d} x^{n}} f \circ g(x)=\sum_{k=1}^{n} f^{(k)}(g(x)) \mathrm{B}_{n, k}\left(g^{\prime}(x), g^{\prime \prime}(x), \ldots, g^{(n-k+1)}(x)\right),
$$

see $[4$, p. 139, Theorem C].

Applying in (2.8) the functions $f(y)=t^{y}$ and $g(x)=u e^{x}-u-1$ gives

$$
\frac{\mathrm{d}^{n} t^{u e^{x}}}{\mathrm{~d} x^{n}}=\sum_{k=1}^{n}(\ln t)^{k} t^{u e^{x}} \mathrm{~B}_{n, k}(\overbrace{u e^{x}, u e^{x}, \ldots, u e^{x}}^{n-k+1}) .
$$

Making use of the formulas (2.6) and (2.7) in (2.9) reveals

$$
\frac{\mathrm{d}^{n} t^{u e^{x}}}{\mathrm{~d} x^{n}}=t^{u e^{x}} \sum_{k=1}^{n} S(n, k) u^{k}(\ln t)^{k} e^{k x} .
$$


Differentiating $n$ times on both sides of (2.4) and considering (2.10) figure out

$$
\frac{\mathrm{d}^{n}}{\mathrm{~d} x^{n}}\left(\frac{x}{e^{x}-1}\right)=\sum_{k=1}^{n} S(n, k) e^{k x} \int_{0}^{\infty} u^{k}\left(\int_{1 / e}^{1}(\ln t)^{k} t^{u e^{x}-u-1} \mathrm{~d} t\right) e^{-u} \mathrm{~d} u .
$$

On the other hand, differentiating $n$ times on both sides of (1.1) gives

$$
\frac{\mathrm{d}^{n}}{\mathrm{~d} x^{n}}\left(\frac{x}{e^{x}-1}\right)=\sum_{k=n}^{\infty} B_{k} \frac{x^{k-n}}{(k-n) !} .
$$

Equating (2.11) and (2.12) and taking the limit $x \rightarrow 0$ discover

$$
\begin{aligned}
B_{n} & =\sum_{k=1}^{n} S(n, k) \int_{0}^{\infty} u^{k}\left(\int_{1 / e}^{1} \frac{(\ln t)^{k}}{t} \mathrm{~d} t\right) e^{-u} \mathrm{~d} u \\
& =\sum_{k=1}^{n} \frac{(-1)^{k}}{k+1} S(n, k) \int_{0}^{\infty} u^{k} e^{-u} \mathrm{~d} u \\
& =\sum_{k=1}^{n} \frac{(-1)^{k} k !}{k+1} S(n, k) .
\end{aligned}
$$

The first proof of Theorem 1.1 is complete.

Second proof. In the book [2, p. 386] and in the papers [3, p. 615] and [12, p. 885], it was given that

$$
\frac{\ln b-\ln a}{b-a}=\int_{0}^{1} \frac{1}{(1-t) a+t b} \mathrm{~d} t
$$

where $a, b>0$ and $a \neq b$. Replacing $a$ by 1 and $b$ by $e^{x}$ yields

$$
\frac{x}{e^{x}-1}=\int_{0}^{1} \frac{1}{1+\left(e^{x}-1\right) t} \mathrm{~d} t
$$

Applying the functions $f(y)=\frac{1}{y}$ and $y=g(x)=1+\left(e^{x}-1\right) t$ in the formula (2.8) and simplifying by (2.6) and (2.7) give

$$
\begin{aligned}
\frac{\mathrm{d}^{n}}{\mathrm{~d} x^{n}}\left(\frac{x}{e^{x}-1}\right) & =\int_{0}^{1} \frac{\mathrm{d}^{n}}{\mathrm{~d} x^{n}}\left[\frac{1}{1+\left(e^{x}-1\right) t}\right] \mathrm{d} t \\
& =\int_{0}^{1} \sum_{k=1}^{n}(-1)^{k} \frac{k !}{\left[1+\left(e^{x}-1\right) t\right]^{k+1}} \mathrm{~B}_{n, k}(\overbrace{t e^{x}, t e^{x}, \ldots, t e^{x}}^{n-k+1}) \mathrm{d} t \\
& =\sum_{k=1}^{n}(-1)^{k} k ! \int_{0}^{1} \frac{t^{k}}{\left[1+\left(e^{x}-1\right) t\right]^{k+1}} \mathrm{~B}_{n, k}(\overbrace{e^{x}, e^{x}, \ldots, e^{x}}^{n-k+1}) \mathrm{d} t \\
& \rightarrow \sum_{k=1}^{n}(-1)^{k} k ! \int_{0}^{1} t^{k} \mathrm{~B}_{n, k}(\overbrace{1,1, \ldots, 1}^{n-k+1}) \mathrm{d} t, \quad x \rightarrow 0 \\
& =\sum_{k=1}^{n}(-1)^{k} k ! S(n, k) \int_{0}^{1} t^{k} \mathrm{~d} t \\
& =\sum_{k=1}^{n}(-1)^{k} \frac{k !}{k+1} S(n, k) .
\end{aligned}
$$


On the other hand, taking the limit $x \rightarrow 0$ in (2.12) leads to

$$
\frac{\mathrm{d}^{n}}{\mathrm{~d} x^{n}}\left(\frac{x}{e^{x}-1}\right)=\sum_{k=n}^{\infty} B_{k} \frac{x^{k-n}}{(k-n) !} \rightarrow B_{n}, \quad x \rightarrow 0 .
$$

The second proof of Theorem 1.1 is thus complete.

Third proof. Let $C T[f(x)]$ be the coefficient of $x^{0}$ in $f(x)$. Then

$$
\begin{aligned}
\sum_{k=1}^{n}(-1)^{k} \frac{k !}{k+1} S(n, k) & =\sum_{k=1}^{n}(-1)^{k} C T\left[\frac{n !}{x^{n}} \frac{\left(e^{x}-1\right)^{k}}{k+1}\right] \\
& =n ! C T\left[\frac{1}{x^{n}} \sum_{k=1}^{\infty}(-1)^{k} \frac{\left(e^{x}-1\right)^{k}}{k+1}\right] \\
& =n ! C T\left[\frac{1}{x^{n}} \frac{\ln \left[1+\left(e^{x}-1\right)\right]-\left(e^{x}-1\right)}{e^{x}-1}\right] \\
& =n ! C T\left[\frac{1}{x^{n}} \frac{x}{e^{x}-1}\right] \\
& =B_{n} .
\end{aligned}
$$

Thus, the formula (1.4) follows.

Fourth proof. It is clear that the equation (1.1) may be rewritten as

$$
\frac{\ln \left[1+\left(e^{x}-1\right)\right]}{e^{x}-1}=\sum_{k=0}^{\infty} B_{k} \frac{x^{k}}{k !} .
$$

Differentiating $n$ times on both sides of (2.15) and taking the limit $x \rightarrow 0$ reveal

$$
\begin{aligned}
B_{n} & =\lim _{x \rightarrow 0} \sum_{k=n}^{\infty} B_{k} \frac{x^{k-n}}{(k-n) !}=\lim _{x \rightarrow 0} \frac{\mathrm{d}^{n}}{\mathrm{~d} x^{n}}\left(\frac{\ln \left[1+\left(e^{x}-1\right)\right]}{e^{x}-1}\right) \\
& =\lim _{x \rightarrow 0} \sum_{k=1}^{n}\left[\frac{\ln (1+u)}{u}\right]^{(k)} \mathrm{B}_{n, k}(\overbrace{e^{x}, e^{x}, \ldots, e^{x}}^{n-k+1}, \quad u=e^{x}-1 \\
& =\lim _{x \rightarrow 0} \sum_{k=1}^{n}\left[\sum_{\ell=1}^{\infty}(-1)^{\ell-1} \frac{u^{\ell-1}}{\ell}\right]^{(k)} \mathrm{B}_{n, k}(\overbrace{e^{x}, e^{x}, \ldots, e^{x}}^{n-k+1} \\
& =\lim _{x \rightarrow 0} \sum_{k=1}^{n}\left[\sum_{\ell=k+1}^{\infty}(-1)^{\ell-1} \frac{(\ell-1) !}{(\ell-k-1) ! \ell} u^{\ell-k-1}\right] \mathrm{B}_{n, k}(\overbrace{e^{x}, e^{x}, \ldots, e^{x}}) \\
& =\sum_{k=1}^{n} \lim _{u \rightarrow 0}\left[\sum_{\ell=k+1}^{\infty}(-1)^{\ell-1} \frac{(\ell-1) !}{(\ell-k-1) ! \ell} u^{\ell-k-1}\right] \lim _{x \rightarrow 0} \mathrm{~B}_{n, k}(\overbrace{e^{x}, e^{x}, \ldots, e^{x}}) \\
& =\sum_{k=1}^{n}(-1)^{k} \frac{k !}{k+1} \mathrm{~B}_{n, k}(\overbrace{1,1, \ldots, 1}^{n-k+1}) \\
& =\sum_{k=1}^{n}(-1)^{k} \frac{k !}{k+1} S(n, k) .
\end{aligned}
$$

The fourth proof of Theorem 1.1 is thus complete. 
Remark 2.1. In [10, p. 1128, Corollary], among other things, it was found that

$$
B_{2 k}=\frac{1}{2}-\frac{1}{2 k+1}-2 k \sum_{i=1}^{k-1} \frac{A_{2(k-i)}}{2(k-i)+1}
$$

for $k \in \mathbb{N}$, where $A_{m}$ is defined by

$$
\sum_{m=1}^{n} m^{k}=\sum_{m=0}^{k+1} A_{m} n^{m}
$$

In $[6$, p. 559] and $[9$, Theorem 2.1], it was collected and recovered that

$$
\left(\frac{1}{e^{x}-1}\right)^{(k)}=(-1)^{k} \sum_{m=1}^{k+1}(m-1) ! S(k+1, m)\left(\frac{1}{e^{x}-1}\right)^{m}, \quad k \in\{0\} \cup \mathbb{N} .
$$

In $[9$, Theorem 3.1], by the identity (2.17), it was obtained that

$$
\begin{aligned}
B_{2 k}=1+\sum_{m=1}^{2 k-1} \frac{S(2 k+1, m+1) S(2 k, 2 k-m)}{\left(\begin{array}{c}
2 k \\
m
\end{array}\right)} & \\
& -\frac{2 k}{2 k+1} \sum_{m=1}^{2 k} \frac{S(2 k, m) S(2 k+1,2 k-m+1)}{\left(\begin{array}{c}
2 k \\
m-1
\end{array}\right)}, \quad k \in \mathbb{N} .
\end{aligned}
$$

In [14, Theorem 1.4], among other things, it was presented for $k \in \mathbb{N}$ that

$$
B_{2 k}=\frac{(-1)^{k-1} k}{2^{2(k-1)}\left(2^{2 k}-1\right)} \sum_{i=0}^{k-1} \sum_{\ell=0}^{k-i-1}(-1)^{i+\ell}\left(\begin{array}{c}
2 k \\
\ell
\end{array}\right)(k-i-\ell)^{2 k-1} .
$$

Recently, a new formula

$$
B_{n}=\sum_{i=0}^{n}(-1)^{i} \frac{\left(\begin{array}{c}
n+1 \\
i+1
\end{array}\right)}{\left(\begin{array}{c}
n+i \\
i
\end{array}\right)} S(n+i, i)
$$

for $n \in \mathbb{N}$ was discovered in the preprint [13].

Remark 2.2. The identities in (2.17) have been generalized and applied in [8, 15].

Remark 2.3. This paper is a slightly revised version of the preprint [7].

Acknowledgements. The authors thank Professor Doron Zeilberg in USA for his reminding of the books $[5,6]$ and sketching the third proof in an e-mail on October 10, 2013. Due to this, the authors find that the formula (1.4) originated from the uneasily-found literature [11] and was listed as an incidental consequence of an answer to an exercise in [5, p. 536] and [6, p. 560].

\section{REFERENCES}

[1] M. Abramowitz and I. A. Stegun (Eds), Handbook of Mathematical Functions with Formulas, Graphs, and Mathematical Tables, National Bureau of Standards, Applied Mathematics Series 55, 9th printing, Washington, 1970. 2

[2] P. S. Bullen, Handbook of Means and Their Inequalities, Mathematics and its Applications, Volume 560, Kluwer Academic Publishers, Dordrecht-Boston-London, 2003. 3

[3] B. C. Carlson, The logarithmic mean, Amer. Math. Monthly 79 (1972), 615-618. 3

[4] L. Comtet, Advanced Combinatorics: The Art of Finite and Infinite Expansions, Revised and Enlarged Edition, D. Reidel Publishing Co., Dordrecht and Boston, 1974. 2 
[5] R. L. Graham, D. E. Knuth, and O. Patashnik, Concrete Mathematics-A Foundation for Computer Science, Addison-Wesley Publishing Company, Advanced Book Program, Reading, MA, 1989. 1, 5

[6] R. L. Graham, D. E. Knuth, and O. Patashnik, Concrete Mathematics-A Foundation for Computer Science, 2nd ed., Addison-Wesley Publishing Company, Reading, MA, 1994. 1, 5

[7] B.-N. Guo and F. Qi, Alternative proofs of a formula for Bernoulli numbers in terms of Stirling numbers, available online at http://arxiv.org/abs/1401.4257. 5

[8] B.-N. Guo and F. Qi, Explicit formulae for computing Euler polynomials in terms of Stirling numbers of the second kind, J. Comput. Appl. Math. (2015), in press; Available online at http://dx.doi.org/10.1016/j.cam.2014.05.018. 5

[9] B.-N. Guo and F. Qi, Some identities and an explicit formula for Bernoulli and Stirling numbers, J. Comput. Appl. Math. 255 (2014), 568-579; Available online at http://dx.doi. org $/ 10.1016 / \mathrm{j} . \mathrm{cam} .2013 .06 .020 .5$

[10] S.-L. Guo and F. Qi, Recursion formulae for $\sum_{m=1}^{n} m^{k}$, Z. Anal. Anwendungen 18 (1999), no. 4, 1123-1130; Available online at http://dx.doi.org/10.4171/ZAA/933. 5

[11] B. F. Logan, Polynomials related to the Stirling numbers, AT\&T Bell Laboratories Internal Technical Memorandum, August 10, 1987. 5

[12] E. Neuman, The weighted logarithmic mean, J. Math. Anal. Appl. 188 (1994), no. 3, 885-900; Available online at http://dx.doi.org/10.1006/jmaa.1994.1469. 3

[13] F. Qi, An explicit formula for Bernoulli numbers in terms of Stirling numbers of the second kind, available online at http://arxiv.org/abs/1401.4255. 5

[14] F. Qi, Explicit formulas for derivatives of tangent and cotangent and for Bernoulli and other numbers, available online at http://arxiv.org/abs/1202.1205. 5

[15] A.-M. Xu and Z.-D. Cen, Some identities involving exponential functions and Stirling numbers and applications, J. Comput. Appl. Math. 260 (2014), 201-207; Available online at http://dx.doi.org/10.1016/j.cam.2013.09.077. 5

(Guo) School of Mathematics and Informatics, Henan Polytechnic University, Jiaozuo City, Henan Province, 454010, China

E-mail address: bai.ni.guo@gmail.com, bai.ni.guo@hotmail.com

(Qi) School of Mathematics and Informatics, Henan Polytechnic University, Jiaozuo City, Henan Province, 454010, China

E-mail address: qifeng618@gmail.com, qifeng618@hotmail.com, qifeng618@qq.com

$U R L:$ http://qifeng618. wordpress . com 